\title{
Oncogenic effects of WNT5A in Epstein-Barr virus-associated nasopharyngeal carcinoma
}

\author{
LEE FAH YAP ${ }^{1}$, MUNIRAH AHMAD ${ }^{2}$, MUHAMMAD MAMDUH AHMAD ZABIDI $^{3}$, TAI LIN CHU $^{2}$, \\ SAN JIUN CHAI ${ }^{3}$, HUI MIN LEE ${ }^{1}$, PAUL VEY HONG LIM ${ }^{4}$, WENBIN WEI ${ }^{5}$, CHRISTOPHER DAWSON $^{5}$, \\ SOO-HWANG TEO ${ }^{3}$ and ALAN SOO BENG KHOO ${ }^{2}$ \\ ${ }^{1}$ Department of Oral Biology and Biomedical Sciences and Oral Cancer Research and Coordinating Centre, \\ Faculty of Dentistry, University of Malaya, Kuala Lumpur; ${ }^{2}$ Molecular Pathology Unit, Cancer Research Centre, \\ Institute for Medical Research, Kuala Lumpur; ${ }^{3}$ Cancer Research Initiatives Foundation, Selangor; \\ ${ }^{4}$ Tung Shin Hospital, Kuala Lumpur, Malaysia; ${ }^{5}$ School of Cancer Sciences, \\ University of Birmingham, Birmingham, UK
}

Received January 6, 2014; Accepted February 27, 2014

DOI: 10.3892/ijo.2014.2342

\begin{abstract}
The molecular events that drive the progression of Epstein-Barr virus (EBV)-associated nasopharyngeal carcinoma (NPC) are still to be elucidated. Here, we report for the first time the pathogenic significance of an NPC-associated gene, wingless-type MMTV integration site family, member 5A (WNT5A) and the contribution of EBV to its expression. WNT5A is a representative Wnt protein that activates non-canonical Wnt signalling. With regard to its role in carcinogenesis, there is conflicting evidence as to whether WNT5A has a tumour-promoting or tumour-suppressive role. We show that WNT5A is upregulated in primary NPC tissue samples. We also demonstrate that WNT5A expression was dramatically increased in NPC cell lines expressing the EBV-encoded LMP2A gene, suggesting that this EBV-encoded latent gene is responsible for upregulating WNT5A in NPC. In addition, in vitro WNT5A overexpression promotes the proliferation, migration and invasion of NPC cells. Our results not only reveal pro-tumorigenic effects of WNT5A in NPC but also suggest that WNT5A could be an important therapeutic target in patients with EBV-associated disease.
\end{abstract}

\section{Introduction}

Nasopharyngeal carcinoma (NPC) is particularly prevalent in southern China and Southeast Asia (1). Radiotherapy is

Correspondence to: Dr Lee Fah Yap, Department of Oral Biology and Biomedical Sciences, Level 9, Postgraduate and Research Tower, Faculty of Dentistry, University of Malaya, 50603 Kuala Lumpur, Malaysia

E-mail: yapleefah@um.edu.my

Key words: nasopharyngeal carcinoma, Epstein-Barr virus, winglesstype MMTV integration site family, member 5A, non-canonical Wnt pathway effective against early stage NPC; however, over $70 \%$ of cases present with late stage diseases and only $10-40 \%$ of these patients survive more than 5 years (2-4). Currently the mainstay of treatment for locoregional advanced cases of NPC is concurrent chemoradiotherapy. Unfortunately, undesirable complications frequently occur after treatment because of the location of the tumour at the base of the skull, closely surrounded by, and in close proximity to, many vital structures that result in high morbidity and poor quality of life (5). Unlike other head and neck cancers, NPC is consistently associated with Epstein-Barr virus (EBV) infection (6). EBV latent gene expression in NPC is restricted to EBNA1, BARF1, variable expression of LMP1 and LMP2A, and consistent expression of the non-coding EBER1/2 RNAs and BARTs, a family of viral microRNAs. The molecular events that drive the progression of NPC, including the exact contribution of EBV to the pathogenesis of NPC, are still to be elucidated.

The Wnt signalling pathways have historically been divided into two classes: namely the canonical and non-canonical pathways. The canonical signalling pathway induces the nuclear accumulation and transcriptional activation of $\beta$-catenin. It is the most intensively studied Wnt pathway implicated in cancer development by promoting cancer cell proliferation and migration (7). By contrast, the non-canonical pathway is essentially an umbrella term for all Wnt-activated cellular signalling pathways that do not promote $\beta$-catenin-mediated transcription. The non-canonical and planar cell polarity (PCP) pathways promote calcium mobilization and activate downstream pathways involved in cell motility and metastasis. However, emerging evidence suggests that these pathways are not as autonomous as originally thought and there may be cross-talk between these two pathways (8). WNT5A is a representative of Wnt protein that activates non-canonical Wnt signalling, it can, under certain circumstances, signal through the canonical pathway (8). A number of studies indicates that WNT5A has a tumour-suppressing effect with reduced expression being reported in colorectal cancer $(9,10)$, neuroblastoma (11), ductal breast cancer $(12,13)$ and leukemias (14-16). Conversely, 
some studies have reported overexpression of WNT5A in melanoma (17), breast cancer cells (18), gastric cancer (19), pancreatic cancer (20), non-small-cell lung cancer (21) and prostate cancer (22), indicating that WNT5A may function as an oncogene in these tumours. Collectively, these data suggest that WNT5A can function as either a tumour suppressor gene or an oncogene depending on the cancer type, and it is possible that interactions between the canonical and non-canonical pathways may explain these discrepancies.

Using expression microarrays, we have previously identified WNT5A as one of the upregulated genes in EBV-positive primary NPC tumours compared with cancer-free nasopharyngeal tissue samples (23). This observation is supported by evidence showing that WNT5A protein is overexpressed in primary NPC tissues $(24,25)$. However, the functional role of WNT5A in NPC and the contribution of EBV to its deregulation have not been investigated. In the present study, the upregulation of WNT5A was further validated in NPC tissues and a dramatic increase in WNT5A expression was observed in LMP2A-expressing NPC cell lines, indicating LMP2A contributes to the upregulation of WNT5A in NPC. Ectopic expression of WNT5A in NPC cell lines significantly promotes cell proliferation, migration and invasion. These data suggest that WNT5A appears to have tumour-promoting activity in EBV-associated NPC.

\section{Materials and methods}

Cell lines and tissue samples. The cell lines used in this study included: NP69 and NP460, immortalised nasopharyngeal epithelial cell lines; eight NPC-derived cell lines, of which seven were EBV negative (TW01, TW04, HONE1, SUNE1, HK1, CNE1 and CNE2) and one of which was EBV-positive (C666-1). NP69, NP460 and seven EBV-negative cell lines were a kind gift from Professor S.W. Tsao (Department of Anatomy, University of Hong Kong, Hong Kong, China), and C666-1 was kindly provided by Professor K.W. Lo (Prince of Wales Hospital, Hong Kong, China). NPC cell lines stably expressing LMP2A have been described previously $(26,27)$ and similar protocols were used to generate cells expressing EBNA1 and LMP1 (28).

Snap-frozen nasopharyngeal biopsies from 16 patients were included in the quantitative real-time PCR analysis: 14 with undifferentiated EBER-positive NPC, and two with histologically normal nasopharynx epithelial cells, with no evidence of malignancy and EBER-negative. All tissue samples were collected from Tung Shin Hospital, Kuala Lumpur, Malaysia. This study was approved by the Medical Research Ethics Committee of the Ministry of Health, Malaysia (KKM/NIHSEC/08/0804/MRG-IMR).

Quantitative real-time $P C R$ and semi-quantitative PCR. Total RNA was extracted using RNeasy mini kit (Qiagen, Manchester, UK) and subjected to reverse transcription using oligo (dT) primer and Superscript II (Invitrogen, Carlsbad, CA, USA). Quantitative real-time PCR (Q-PCR) was performed in triplicate using ABI Prism 7000 Sequence Detection System and TaqMan Gene Expression Assays (WNT5A: Hs00998537_m1; Applied Biosystems, Foster City, CA, USA). In parallel, GAPDH was amplified in the same reaction to serve as an internal control for normalization. Fold changes in gene expression between control and samples were measured using the comparative threshold cycle method $(\Delta \Delta \mathrm{Ct})$. Semi-quantitative PCR was performed to examine the WNT5A mRNA level in a panel of 16 human normal organs using the Human MTC ${ }^{\text {тм }}$ Panel I \& II (Clontech, Mountain View, CA, USA). The primers for WNT5A were 5'-CATTATGGGCTCAAATAGAAAGAAGA-3' and 5'-AAAGAGCTAGGGTAGGCAACTAAAACT-3'.

Western blot analysis. Cells were lysed in ice-cold RIPA buffer containing protease inhibitor cocktail (Roche, Indianapolis, IN, USA) and the extracted protein was subjected to western blot analysis. The primary antibody used was WNT5A (1:500, R\&D Systems, Minneapolis, MN, USA) and the secondary antibody was HRP-conjugated anti-goat $\operatorname{IgG}(1: 5,000$, Chemicon, Temecula, CA, USA). The protein bands were detected with chemiluminescent reagent, Perkin Elmer plus (Perkin-Elmer, Waltham, MA, USA) or West Femto (Pierce, Rockford, IL, USA).

Retroviral transduction and establishment of stable cell lines. The recombinant retroviral vectors pLNC-WNT5A or pLNCX alone were used to establish TWO4 and HONE1 NPC cells stably overexpressing WNT5A and the vector control. Briefly, the retroviral vectors were transfected into retroviral packaging cell lines, Phoenix Ampho using Lipofectamine 2000 (Invitrogen). At $48 \mathrm{~h}$ post-transfection, the retroviral-containing supernatant was centrifuged and filtered through $0.45 \mu \mathrm{m}$ syringe filter (Millipore, Billerica, MA, USA). The viral supernatant was then added to plates cultured with TWO4 or HONE1 cells. Polybrene $(0.8 \mu \mathrm{g} / \mathrm{ml}$; Chemicon) was also added to the culture media. After the transduction $(24 \mathrm{~h})$, cells were selected with $500 \mu \mathrm{g} / \mathrm{ml}$ neomycin. Drug-resistant cells were then pooled and tested for WNT5A expression by western blot analysis.

Cell proliferation assay. Cells $\left(3-6 \times 10^{3}\right)$ were seeded in a 96-well plate in triplicate. Cell proliferation was determined daily by adding $20 \mu \mathrm{l}$ of MTT (thiazolyl blue tetrazolium bromide, Sigma, St. Louis, MO, USA) stock solution $(5 \mathrm{mg} / \mathrm{ml})$ to each well for 4 days. After $4 \mathrm{~h}$ of incubation, medium was removed carefully and $100 \mu \mathrm{l}$ DMSO was added to dissolve the blue crystals. The absorbance was measured on an ELISA plate reader at a wavelength of $570 \mathrm{~nm}$ with reference wavelength of $630 \mathrm{~nm}$.

Wound healing assay. Cells were grown as a monolayer in 6 -well plates coated with fibronectin $(20 \mu \mathrm{g} / \mathrm{ml})$ and serum starved overnight. Cells were then treated with mitomycin $\mathrm{C}$ $(25 \mu \mathrm{g} / \mathrm{ml})$ for $2 \mathrm{~h}$ to inhibit cell proliferation. A sterile pipette tip was used to scratch a wound along the centre of the well. A demarcated area of the wound was photographed on an inverted Olympus IX71 microscope at the time of wounding $(0 \mathrm{~h})$ and at various time of wound healing.

Cell migration and invasion assays. Migration and invasion assays were carried out using fibronectin-coated $(10 \mu \mathrm{g} / \mathrm{ml})$ and Matrigel-coated polycarbonate filters (8- $\mu \mathrm{m}$ pore size, Transwell Costar Corning; Corning, NY, USA), respectively, as described previously (29). Cells were plated into the upper chamber and allowed to migrate for $24 \mathrm{~h}$ (for migration assay) or $48 \mathrm{~h}$ (for invasion assay). Cells migrating/invading to the 


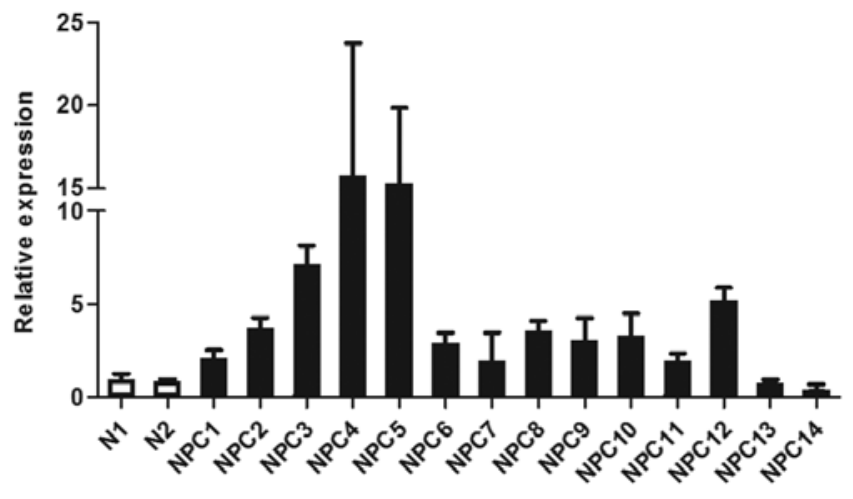

Figure 1. Upregulation of WNT5A expression in EBV-positive NPC. Q-PCR showed that, compared with two biopsies of normal nasopharynx (N1, N2), WNT5A mRNA was significantly upregulated in 12/14 NPC tumours (WNT5A expression of NPC 13 and NPC 14 also did not show an increase in microarray analysis). Shown here are data comparing tumours with the normal sample, in which WNT5A expression level was higher than the control sample, N1. The expression level of N1 was normalized to 1 .

lower chamber were trypsinized and counted on a Casy 1 counter (Schärfe System GmbH, Reutlingen, Germany). Statistical differences between experimental groups were evaluated by Student's t-test.

\section{Results}

Overexpression of WNT5A in EBV-positive NPC. Using microarray analysis, we previously demonstrated upregulation of WNT5A in 20/25 (80\%) of EBV-positive NPC tissue samples, while expression in normal nasopharyngeal epithelium was low or absent (23). In agreement with the microarray data, Q-PCR showed that WNT5A mRNA levels were elevated in 12 NPC tissue samples available for analysis when compared to two non-malignant controls (Fig. 1). Two NPC samples (NPC13 and NPC14) that did not overexpress WNT5A in the microarray analysis also did not show an elevated level of WNT5A by Q-PCR, confirming the validity of the microarray data.

LMP2A upregulates WNT5A expression. The expression of WNT5A was heterogeneous in NPC cell lines. Nonetheless, particularly high levels of WNT5A expression were observed in the only EBV-positive cell line, C666-1, compared to the a panel of EBV-negative cell lines, which included seven NPC cell lines (CNE1, CNE2, HONE1, HK1, SUNE1, TW01, TW04) and two immortalised nasopharyngeal epithelial cell lines (NP460, NP69; Fig. 2A).

To further investigate which EBV gene is responsible for the upregulation of WNT5A, the expression of WNT5A was examined in HONE1 cells independently transfected with three EBV latent genes (EBNA1, LMP1 and LMP2A). The results showed that levels of WNT5A mRNA were significantly upregulated in cells transfected with EBNA1 and LMP2A $(\mathrm{p}<0.01$; Fig. 2B). Due to the robust upregulation of WNT5A in LMP2A-expressing cells, we further examined the expression of WNT5A in another NPC cell line, CNE2, transfected with LMP2A. We confirmed that LMP2A stimulated WNT5A expression in NPC cells ( $p<0.01$; Fig. 2 C).
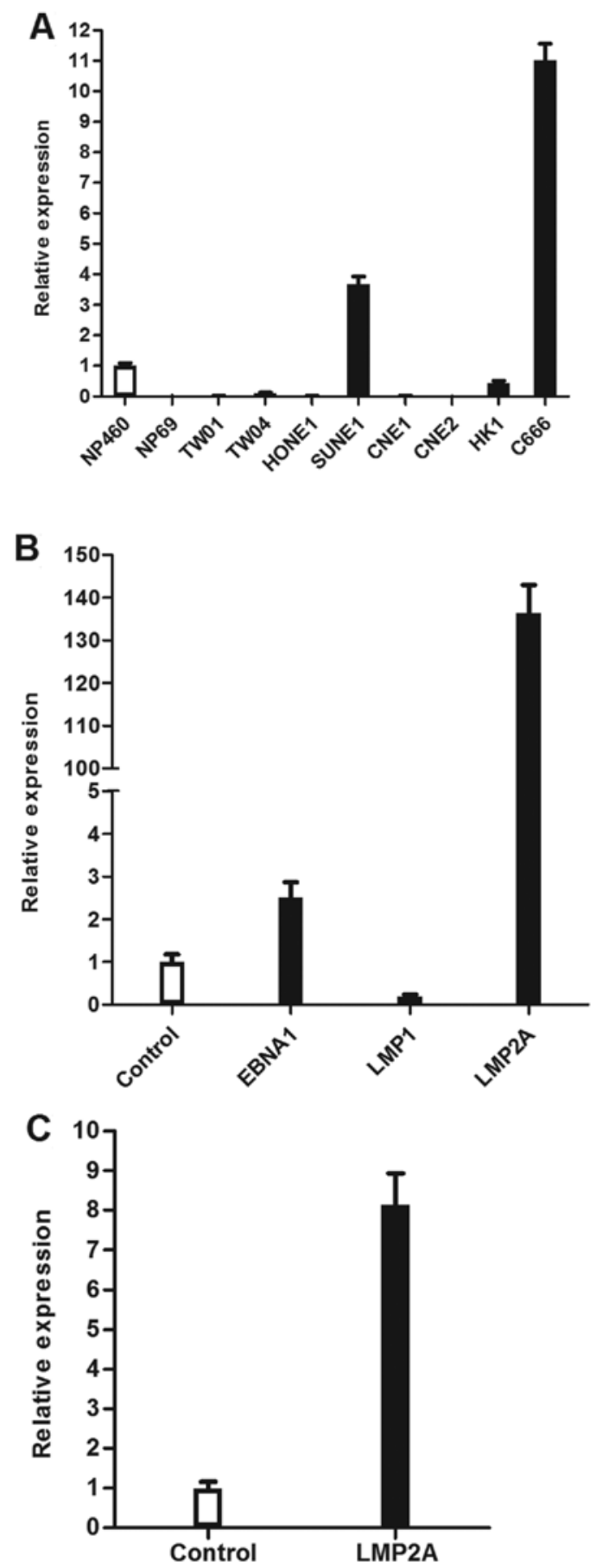

Figure 2. EBV infection stimulates the expression of WNT5A. (A) In NPC cell lines, Q-PCR analysis showed that the only EBV-positive cell line, C666-1, had markedly increased levels of WNT5A when compared to a panel of EBV-negative cell lines. Shown here are data comparing cell lines with NP460 cells, an immortalised nasopharyngeal epithelial cell line. (B) Q-PCR analysis showed that the expression of WNT5A transcripts was significantly increased in HONE1 cells expressing EBV-encoded EBNA1 and LMP2A (p<0.01). (C) The ability of LMP2A to stimulate the expression of WNT5A was further confirmed in CNE2 cells expressing LMP2A $(\mathrm{p}<0.01)$. Data are expressed as the relative expression between the cells transfected with EBV latent genes and their respective controls. The expression levels of the controls were normalised to 1 .

WNT5A promotes cell growth. To investigate the functional role of WNT5A in NPC, two NPC cell lines which expressed low levels of endogenous WNT5A, HONE1 and TW04, were stably transduced with recombinant retroviral vector $\mathrm{pLNCX}$ 


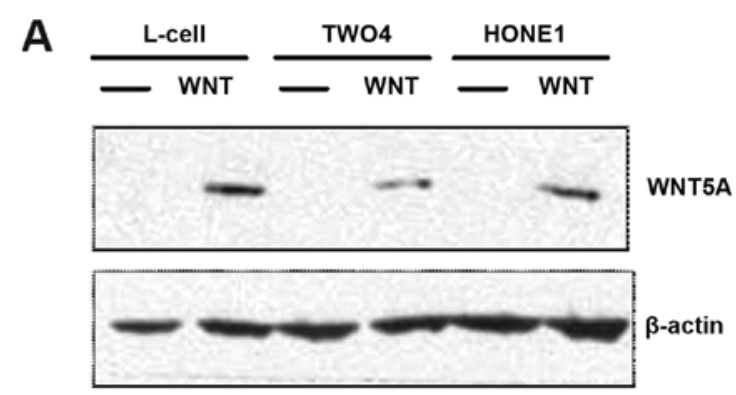

B
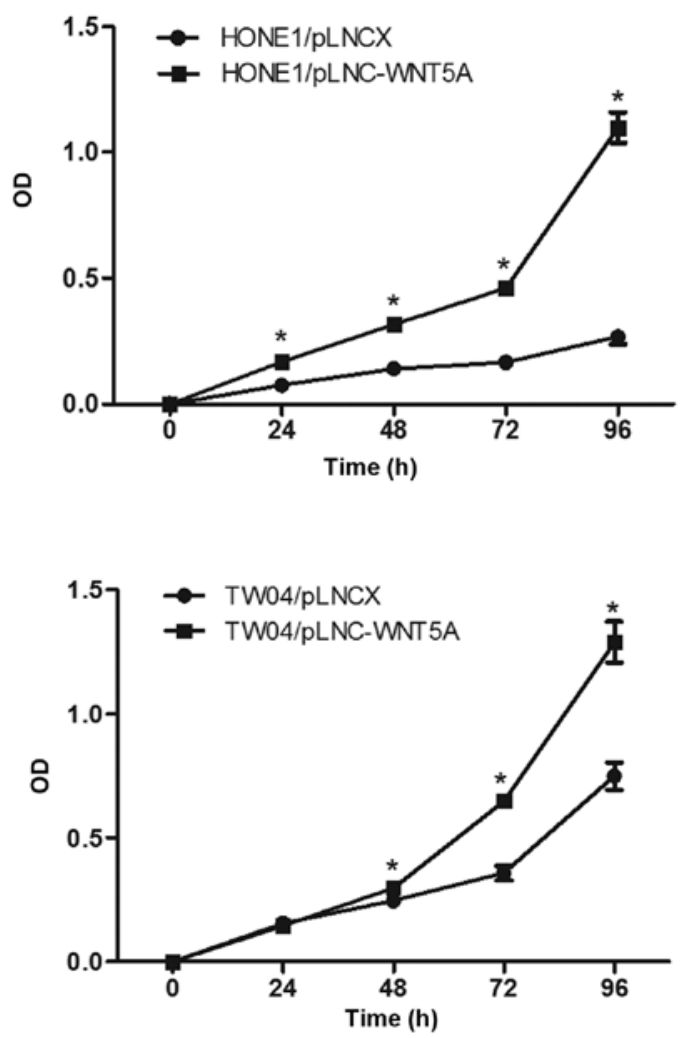

Figure 3. WNT5A promotes cell proliferation. (A) NPC cell lines transduced with pLNC-WNT5A or vector alone were analysed by western blot analysis. L-cells stably expressing WNT5A were used as a positive control. (B) The contribution of WNT5A in cell growth was examined by MTT colorimetric assays for 4 days after cell plating. The results showed that HONE1 and TW04 cells expressing WNT5A grew significantly faster than vector control cells ( $\mathrm{p} p<0.01)$. All experiments were carried out in triplicate and repeated at least 3 times.

containing the WNT5A cDNA (designated pLNC-WNT5A) or with the vector alone (designated pLNC). The expression of WNT5A protein in these two cell lines was confirmed by western blot analysis (Fig. 3A).

We next examined the effect of ectopic expression of WNT5A on the growth of NPC cells using MTT assays. As shown in Fig. 3B, HONE1 and TW04 cells stably expressing WNT5A grew significantly faster than the vector control cells ( $<<0.01$ ), indicating that the WNT5A promotes cell proliferation.

WNT5A promotes cell migration and invasion. To investigate whether WNT5A functions to promote cell migration in NPC, a wound-healing assay was performed using HONE1 and
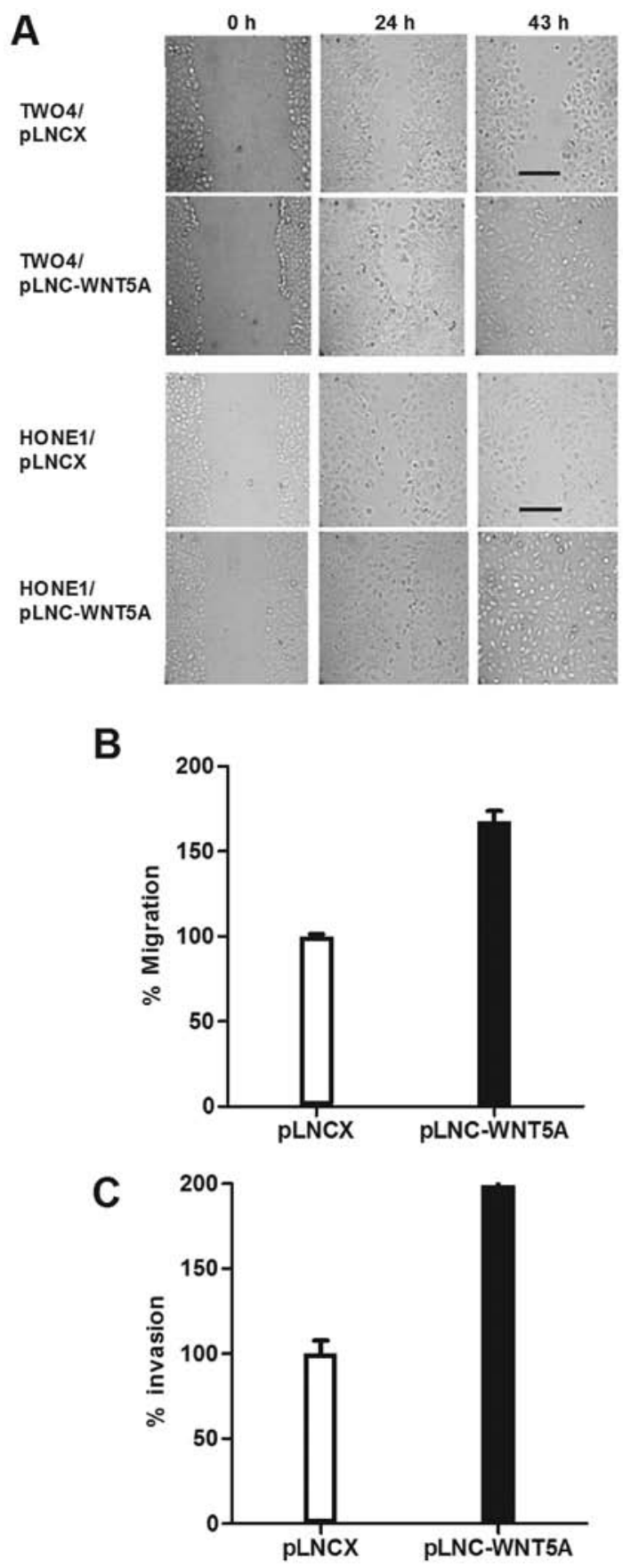

Figure 4. WNT5A promotes cell migration and invasion. (A) A wound healing assay showed that the motility of both the TW04 and HONE1 cells stably expressing WNT5A was enhanced compared to vector control cells. (B) The migration ability of HONE1 transfected cells was further examined in fibronectin-coated Transwell migration assays. Data are expressed as mean percentage of cells migrating $\pm \mathrm{SD}$ and the results are expressed relative to migration of vector only transfected cells (100\%). The migration of HONE1 cells expressing WNT5A was significantly increased compared to control cells $(\mathrm{p}<0.01)$. (C) The invasiveness of the HONE1 transfected cells was examined in a Matrigel assay. Data are expressed as mean percentage of cells invading \pm SD and the results are expressed relative to invasion of vector only transfected cells (100\%). The invasiveness of HONE1 cells expressing WNT5A was significantly increased compared to control cells $(\mathrm{p}=0.01)$.

TW04 transfected cells. Compared to the vector controls, an increase in cell motility was observed in cells expressing WNT5A (Fig. 4A). This result was further confirmed using standard Transwell assays which showed that migration of 


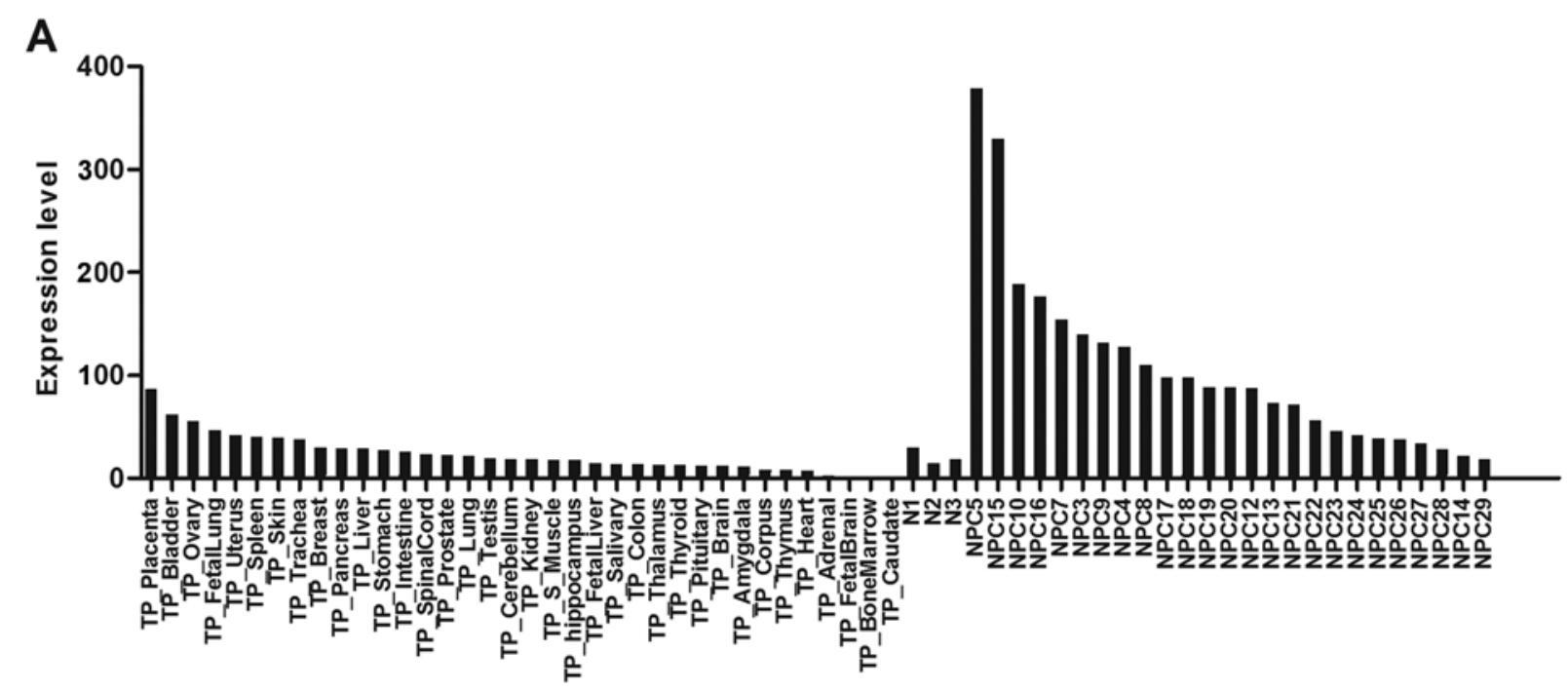

B

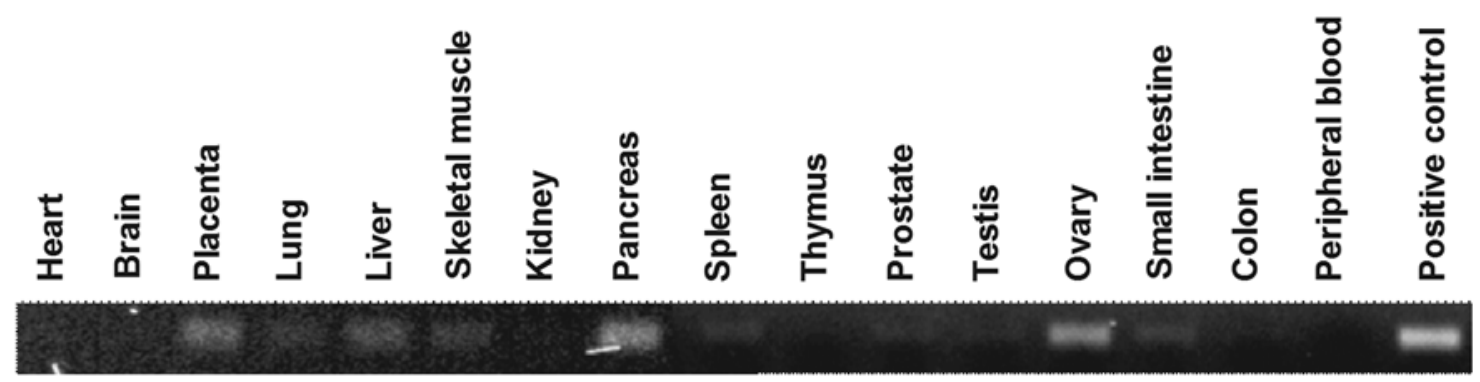

Figure 5. Low expression of WNT5A in normal human organs. (A) A comparison between our NPC microarray analysis and a published microarray study using 36 normal human organs revealed that the WNT5A level in NPC was significantly higher than that in a wide range of normal organs with exception of placenta, ovary and bladder. N1, N2, N3 are non-malignant nasopharyngeal tissues. (B) The low expression of WNT5A expression in human normal organs was validated in a panel of 16 human normal organs using Multiple cDNA Panels (BD BioScience). Consistent with the microarray data in general, the expression of WNT5A was low in various normal organs but was readily detectable in placenta, pancreas and ovary. The cDNA of C666-1 cells served as a positive control for the PCR reaction.

HONE1 cells expressing WNT5A was significantly enhanced $(\mathrm{p}<0.01$; Fig. 4B).

We next examined the effect of WNT5A on the invasiveness of NPC cells using Matrigel invasion assays. WNT5A-transfected HONE1 cells were 2 times more invasive than the vector controls ( $\mathrm{p}=0.01$; Fig. $4 \mathrm{C}$ ).

\section{Discussion}

NPC is the most common cancer arising in nasopharynx. Despite recent advances in treatment regimens, NPC patients continue to have generally poor prognoses due to unwanted side-effects and a subset of tumours is resistant to radiotherapy and chemotherapy. The identification of genes differentially expressed between normal and malignant cells may lead to the identification of biomarkers and novel therapeutic targets for this disease. We have previously shown that WNT5A is overexpressed in NPC relative to cancer-free controls (23). WNT5A is one of the most highly investigated Wnt proteins that activate non-canonical Wnt signalling. At present, the role of WNT5A in human cancer is controversial and the discrepancy may be attributable to differences in receptor context or cell context of cancer cells. Although aberrant Wnt signalling has been implicated in the development of NPC, these early studies mainly focused on the canonical $\mathrm{Wnt} / \beta$-catenin pathway which is often activated in human cancers (30). Recently, immunohistochemical data have shown that the WNT5A protein is overexpressed in primary NPC tissues $(24,25)$, suggesting a dysregulation of non-canonical pathway in NPC. However, information regarding the biological role of WNT5A in the pathogenesis of NPC or the mechanisms of its overexpression has not been explored. It should be noted that a comparison between our NPC microarray data and a published microarray study using 36 normal human organs (31) revealed that the levels of WNT5A expression in NPC was higher than that observed in a wide range of normal organs, with exception of placenta, ovary and bladder (Fig. 5A). Similarly, we showed that the expression of WNT5A was low in various normal human organs using Multiple cDNA Panels (BD BioSciences, Franklin Lakes, NJ, USA) by PCR analysis (Fig. 5B). These observations suggest that WNT5A or its downstream signalling events could be potentially useful targets for treatment of NPC.

In this study, we further confirmed the upregulation of WNT5A mRNA in primary NPC tissue samples by QPCR, supporting the hypothesis that WNT5A exhibits an oncogenic effect in NPC. The strong etiological link between EBV infection and NPC is well recognised (6). Most, if not all, NPC cases in endemic regions, such as Malaysia, are associated 
with EBV infection $(23,32)$. Interestingly, in the present study, very high levels of WNT5A expression were observed in the only EBV-positive NPC cell line, C666.1, compared to a panel of EBV-negative epithelial cells. Further, we found that the levels of WNT5A were dramatically increased in HONE1 and CNE2 cells stably expressing the EBV-encoded oncogene LMP2A. LMP2A mRNA is regularly detected in NPC and when expressed in certain immortalized epithelial cell lines, it can induce anchorage-independent growth, enhance cell adhesion and cell motility, and inhibit epithelial cell differentiation (33). Previous studies indicate that LMP2A can modulate the canonical Wnt pathway in EBV-infected epithelial cells (34-36). Here, we report, for the first time, that EBV infection, particularly LMP2A, may also play a major role in inducing an aberrant non-canonical Wnt signalling in NPC.

Uncontrolled cell growth and tissue invasion/metastasis are hallmarks of cancer cells, and it is well established that EBV-positive NPC is a highly metastatic cancer (37). In tumours that express elevated levels of WNT5A, WNT5A functions as an oncogene by promoting cell proliferation and migration/invasion $(20,38,39)$. Similarly, we showed that WNT5A promotes cell growth and migration/invasion of NPC cells, contributing to the acquisition of a highly motile and invasive phenotype, a phenotype that is consistent with the highly aggressive behaviour of NPC cells. In melanoma, WNT5A exerted its pro-migratory and invasion effects by activating PKC (39). This data was supported by a subsequent study in gastric cancer in which WNT5A was found to promote migration of cancer cells by stimulating focal adhesion kinase (FAK) and Rac through the activation of PKC and JNK (19). A recent study also showed that WNT5A activated JNK through PKD to promote aggressiveness of prostate cancer (40). These studies also demonstrated that the expression of WNT5A and $\beta$-catenin in cancer cells was mutually exclusive, suggesting WNT5A primarily functions through the non-canonical planar cell polarity and Wnt calcium signalling pathways.

Although WNT5A may inhibit the activation of $\beta$-catenin-mediated transcription, there is evidence to suggest that in the presence of specific forms of receptors, WNT5A could stimulate cancer-promoting canonical Wnt signalling pathway in certain cell types $(41,42)$. This phenomenon has been shown in pancreatic cancer that WNT5A mediated its pro-invasive effects through $\beta$-catenin/TCF-dependent pathway (20). We have recently generated a compendium of potential biomarkers for NPC by systematically comparing the genes that are differentially expressed between NPC and cancer-free controls from published microarray studies $(23,25,43-45)$. In concordance with the previous studies reporting the dysregulation of $\mathrm{Wnt} / \beta$-catenin signalling in NPC, overexpression of a number of genes involved in this pathway were commonly shown in independent datasets (Table I). These data suggest that further studies are warranted to elucidate the signalling events downstream WNT5A in NPC.

In summary, we report that WNT5A is overexpressed in primary NPC tissues, where it may function to promote tumour growth, migration and invasion. Furthermore, we show that LMP2A induces the transcription of WNT5A. Taken together, these results not only demonstrate pro-tumorigenic effects of WNT5A in NPC, but also that patients with EBV-associated NPC could potentially benefit from the therapeutic targeting of this molecule.
Table I. Genes involved in the Wnt/ $\beta$-catenin signalling pathway commonly identified to be upregulated in primary NPC tissues in microarray studies.

\begin{tabular}{|c|c|}
\hline Gene symbol & Gene name \\
\hline AKT3 & $\begin{array}{l}\text { v-akt murine thymoma viral oncogene } \\
\text { homolog } 3 \text { (protein kinase B, gamma) }\end{array}$ \\
\hline BIRC5 & Baculoviral IAP repeat-containing 5 \\
\hline CCND2 & Cyclin D2 \\
\hline CD44 & CD44 molecule (Indian blood group) \\
\hline CDC42EP3 & $\begin{array}{l}\text { CDC42 effector protein } \\
\text { (Rho GTPase binding) } 3\end{array}$ \\
\hline CLDN1 & Claudin 1 \\
\hline DKK1 & Dickkopf homolog 1 (Xenopus laevis) \\
\hline DVL3 & Dishevelled, dsh homolog 3 (Drosophila) \\
\hline EPHB1 & EPH receptor B1 \\
\hline FZD5 & Frizzled homolog 5 (Drosophila) \\
\hline FZD6 & Frizzled homolog 6 (Drosophila) \\
\hline FZD7 & Frizzled homolog 7 (Drosophila) \\
\hline GREM1 & Gremlin 1 \\
\hline JAG2 & Jagged 2 \\
\hline JUN & Jun proto-oncogene \\
\hline LGR5 & $\begin{array}{l}\text { Leucine-rich repeat-containing } \\
\text { G protein-coupled receptor } 5\end{array}$ \\
\hline LEF1 & Lymphoid enhancer-binding factor 1 \\
\hline LRP4 & $\begin{array}{l}\text { Low density lipoprotein } \\
\text { receptor-related protein } 4\end{array}$ \\
\hline LRPPRC & Leucine-rich PPR-motif containing \\
\hline MMP9 & Matrix metallopeptidase 9 \\
\hline PRKAB2 & $\begin{array}{l}\text { Protein kinase, AMP-activated, } \\
\text { beta } 2 \text { non-catalytic subunit }\end{array}$ \\
\hline PRKCI & Protein kinase $\mathrm{C}$, iota \\
\hline PTTG1 & Pituitary tumor-transforming 1 \\
\hline SOX2 & SRY (sex determining region Y)-box 2 \\
\hline TCF12 & Transcription factor 12 \\
\hline TCF3 & Transcription factor 3 \\
\hline TCF7L2 & Transcription factor 7-like 2 \\
\hline VCAN & Versican \\
\hline VEGFA & Vascular endothelial growth factor A \\
\hline
\end{tabular}

\section{Acknowledgements}

This study is supported by the University of Malaya (UM.C/625/1/HIR/MOHE/DENT/23), Ministry of Health, Malaysia (JPP-IMR 06-056) and Cancer Research Initiatives Foundation (06-061). We thank the Director General of Health of Malaysia for his permission to publish this article and the Director of the Institute for Medical Research, Kuala Lumpur, for her support. We wish to thank P. Siti Rohana, A.S. Roslinda and other staff of the Molecular Pathology Unit, IMR for their assistance. We also wish to thank A.M.C. Brown (Cornell University) for the pLNC-WNT5A. 


\section{References}

1. Yu MC and Yuan JM: Epidemiology of nasopharyngeal carcinoma. Semin Cancer Biol 12: 421-429, 2002.

2. Qin DX, Hu YH, Yan JH, et al: Analysis of 1379 patients with nasopharyngeal carcinoma treated by radiation. Cancer 61: $1117-1124,1988$.

3. Pua KC, Khoo AS, Yap YY, et al: Nasopharyngeal Carcinoma Database. Med J Malaysia 63 (Suppl C): 59-62, 2008.

4. Lee AW, Poon YF, Foo W, et al: Retrospective analysis of 5037 patients with nasopharyngeal carcinoma treated during 1976-1985: overall survival and patterns of failure. Int J Radiat Oncol Biol Phys 23: 261-270, 1992.

5. Agulnik $M$ and Epstein JB: Nasopharyngeal carcinoma: current management, future directions and dental implications. Oral Oncol 44: 617-627, 2008.

6. Pathmanathan R, Prasad U, Sadler R, Flynn K and Raab-Traub N: Clonal proliferations of cells infected with Epstein-Barr virus in preinvasive lesions related to nasopharyngeal carcinoma N Engl J Med 333: 693-698, 1995.

7. Giles RH, van Es JH and Clevers H: Caught up in a Wnt storm: Wnt signaling in cancer. Biochim Biophys Acta 1653: 1-24, 2003.

8. McDonald SL and Silver A: The opposing roles of Wnt-5a in cancer. Br J Cancer 101: 209-214, 2009.

9. Ying J, Li H, Yu J, et al: WNT5A exhibits tumor-suppressive activity through antagonizing the Wnt/beta-catenin signaling, and is frequently methylated in colorectal cancer. Clin Cancer Res 14: 55-61, 2008

10. Dejmek J, Dejmek A, Safholm A, Sjolander A and Andersson T: Wnt-5a protein expression in primary dukes B colon cancers identifies a subgroup of patients with good prognosis. Cancer Res 65: 9142-9146, 2005.

11. Blanc E, Roux GL, Benard J and Raguenez G: Low expression of Wnt-5a gene is associated with high-risk neuroblastoma. Oncogene 24: 1277-1283, 2005.

12. Jonsson M, Dejmek J, Bendahl PO and Andersson T: Loss of Wnt-5a protein is associated with early relapse in invasive ductal breast carcinomas. Cancer Res 62: 409-416, 2002.

13. Dejmek J, Leandersson K, Manjer J, et al: Expression and signaling activity of Wnt-5a/discoidin domain receptor-1 and Syk plays distinct but decisive roles in breast cancer patient survival. Clin Cancer Res 11: 520-528, 2005.

14. Ying J, Li H, Chen YW, Srivastava G, Gao Z and Tao Q: WNT5A is epigenetically silenced in hematologic malignancies and inhibits leukemia cell growth as a tumor suppressor. Blood 110: 4130-4132, 2007.

15. Roman-Gomez J, Jimenez-Velasco A, Cordeu L, et al: WNT5A, a putative tumour suppressor of lymphoid malignancies, is inactivated by aberrant methylation in acute lymphoblastic leukaemia. Eur J Cancer 43: 2736-2746, 2007.

16. Liang H, Chen Q, Coles AH, et al: Wnt5a inhibits B cell proliferation and functions as a tumor suppressor in hematopoietic tissue. Cancer Cell 4: 349-360, 2003.

17. Da Forno PD, Pringle JH, Hutchinson P, et al: WNT5A expression increases during melanoma progression and correlates with outcome. Clin Cancer Res 14: 5825-5832, 2008.

18. Fernandez-Cobo M, Zammarchi F, Mandeli J, Holland JF and Pogo BG: Expression of Wnt5A and Wnt10B in non-immortalized breast cancer cells. Oncol Rep 17: 903-907, 2007.

19. Kurayoshi M, Oue N, Yamamoto H, et al: Expression of Wnt-5a is correlated with aggressiveness of gastric cancer by stimulating cell migration and invasion. Cancer Res 66: 10439-10448, 2006.

20. Ripka S, Konig A, Buchholz M, et al: WNT5A - target of CUTL1 and potent modulator of tumor cell migration and invasion in pancreatic cancer. Carcinogenesis 28: 1178-1187, 2007.

21. Huang CL, Liu D, Nakano J, et al: Wnt5a expression is associated with the tumor proliferation and the stromal vascular endothelial growth factor - an expression in non-small-cell lung cancer. J Clin Oncol 23: 8765-8773, 2005.

22. Wang Q, Williamson M, Bott S, et al: Hypomethylation of WNT5A, CRIP1 and S100P in prostate cancer. Oncogene 26 : 6560-6565, 2007.
23. Bose S, Yap LF, Fung M, et al: The ATM tumour suppressor gene is down-regulated in EBV-associated nasopharyngeal carcinoma. J Pathol 217: 345-352, 2009.

24. Zeng ZY, Zhou YH, Zhang WL, et al: Gene expression profiling of nasopharyngeal carcinoma reveals the abnormally regulated Wnt signaling pathway. Hum Pathol 38: 120-133, 2007.

25. Hu C, Wei W, Chen X, et al: A global view of the oncogenic landscape in nasopharyngeal carcinoma: an integrated analysis at the genetic and expression levels. PLoS One 7: e41055, 2012.

26. Moody CA, Scott RS, Amirghahari N, et al: Modulation of the cell growth regulator mTOR by Epstein-Barr virus-encoded LMP2A. J Virol 79: 5499-5506, 2005.

27. Shah KM, Stewart SE, Wei W, et al: The EBV-encoded latent membrane proteins, LMP2A and LMP2B, limit the actions of interferon by targeting interferon receptors for degradation. Oncogene 28: 3903-3914, 2009.

28. Allen MD, Young LS and Dawson CW: The Epstein-Barr virus-encoded LMP2A and LMP2B proteins promote epithelial cell spreading and motility. J Virol 79: 1789-1802, 2005.

29. Yap LF, Jenei V, Robinson CM, et al: Upregulation of Eps8 in oral squamous cell carcinoma promotes cell migration and invasion through integrin-dependent Racl activation. Oncogene 28: 2524-2534, 2009.

30. Tulalamba W and Janvilisri T: Nasopharyngeal carcinoma signaling pathway: an update on molecular biomarkers. Int J Cell Biol 2012: 594681, 2012.

31. Ge X, Yamamoto S, Tsutsumi S, et al: Interpreting expression profiles of cancers by genome-wide survey of breadth of expression in normal tissues. Genomics 86: 127-141, 2005.

32. Yap YY, Hassan S, Chan M, Choo PK and Ravichandran M: Epstein-Barr virus DNA detection in the diagnosis of nasopharyngeal carcinoma. Otolaryngol Head Neck Surg 136: 986-991, 2007.

33. Dawson CW, Port RJ and Young LS: The role of the EBV-encoded latent membrane proteins LMP1 and LMP2 in the pathogenesis of nasopharyngeal carcinoma (NPC). Semin Cancer Biol 22: 144-153, 2012.

34. Morrison JA and Raab-Traub N: Roles of the ITAM and PY motifs of Epstein-Barr virus latent membrane protein $2 \mathrm{~A}$ in the inhibition of epithelial cell differentiation and activation of \{beta\}-catenin signaling. J Virol 79: 2375-2382, 2005.

35. Morrison JA, Klingelhutz AJ and Raab-Traub N: Epstein-Barr virus latent membrane protein $2 \mathrm{~A}$ activates beta-catenin signaling in epithelial cells. J Virol 77: 12276-12284, 2003.

36. Morrison JA, Gulley ML, Pathmanathan R and Raab-Traub N: Differential signaling pathways are activated in the EpsteinBarr virus-associated malignancies nasopharyngeal carcinoma and Hodgkin lymphoma. Cancer Res 64: 5251-5260, 2004.

37. Hanahan D and Weinberg RA: The hallmarks of cancer. Cell 100: 57-70, 2000 .

38. Yu JM, Jun ES, Jung JS, et al: Role of Wnt5a in the proliferation of human glioblastoma cells. Cancer Lett 257: 172-181, 2007.

39. Weeraratna AT, Jiang Y, Hostetter G, et al: Wnt5a signaling directly affects cell motility and invasion of metastatic melanoma. Cancer Cell 1: 279-288, 2002

40. Yamamoto H, Oue N, Sato A, et al: Wnt5a signaling is involved in the aggressiveness of prostate cancer and expression of metalloproteinase. Oncogene 29: 2036-2046, 2010.

41. Torii K, Nishizawa K, Kawasaki A, et al: Anti-apoptotic action of Wnt5a in dermal fibroblasts is mediated by the PKA signaling pathways. Cell Signal 20: 1256-1266, 2008.

42. Mikels AJ and Nusse R: Purified Wnt5a protein activates or inhibits beta-catenin-TCF signaling depending on receptor context. PLoS Biol 4: e115, 2006.

43. Fang W, Li X, Jiang Q, et al: Transcriptional patterns, biomarkers and pathways characterizing nasopharyngeal carcinoma of Southern China. J Transl Med 6: 32, 2008

44. Shi W, Bastianutto C, Li A, et al: Multiple dysregulated pathways in nasopharyngeal carcinoma revealed by gene expression profiling. Int J Cancer 119: 2467-2475, 2006.

45. Sriuranpong V, Mutirangura A, Gillespie JW, et al: Global gene expression profile of nasopharyngeal carcinoma by laser capture microdissection and complementary DNA microarrays. Clin Cancer Res 10: 4944-4958, 2004. 